\title{
OPPENHEIM'S INEQUALITY AND RKHS
}

\author{
AKIRA YAMADA
}

Abstract. Applying norm inequalities for RKHSs corresponding to the product of reproducing kernels and using the minimal norm of the Nevanlinna interpolation, we give the basic background and essences of the quite famous fundamental inequalities, Oppenheim's inequality, Hadamard's inequality and Schur's inequality on positive semidefinite matrices. In particular, as an application, we determine equality conditions for Oppenheim's inequality and Schur's inequality.

Mathematics subject classification (2010): Primary 46E22; Secondary 15A45.

Keywords and phrases: Oppenheim's inequality, Hadamard's inequality, Schur's inequality, Hadamard product of matrices, positive semidefinite matrices, reproducing kernels.

\section{REFERENCES}

[1] N. Aronszajn, Theory of reproducing kernels, Trans. Amer. Math. Soc. 68 (1950), 337-404. MR $0051437(14,479 \mathrm{c})$

[2] S. Bergman, The kernel function and conformal mapping, revised ed., American Mathematical Society, Providence, R.I., 1970, Mathematical Surveys, No. V. MR 0507701 (58 \#22502)

[3] R. A. Horn And C. R. Johnson, Matrix analysis, Cambridge University Press, Cambridge, 1985. MR 832183 (87e:15001)

[4] A. Oppenheim, Inequalities connected with definite Hermitian forms, J. London Math. Soc. 5, 2 (1930), 114-119.

[5] S. SAitoH, Theory of reproducing kernels and its applications, Pitman Research Notes in Mathematics Series, vol. 189, Longman Scientific \& Technical, Harlow, 1988. MR 983117 (90f:46045)

[6] A. YAMADA, Equality conditions for norm inequalities in reproducing kernel Hilbert spaces, Math. Inequal. Appl. 12, 2 (2009), 377-390. MR 2521393 (2010f:46048)

[7] X.-D. Zhang And C.-X. Ding, The equality cases for the inequalities of Oppenheim and Schur for positive semi-definite matrices, Czechoslovak Math. J. 59(134), 1 (2009), 197-206. MR 2486625 (2010a:15056) 